SELECCIONES MATEMÁTICAS
Universidad Nacional de Trujillo
ISSN: 2411-1783 (Online)
2021; Vol. 8(1): 161-172.

\title{
Validation of simulated precipitation forecasts with the BRAMS model for the La Libertad-Peru region
}

\section{Validación de los pronósticos de precipitación simulados con el modelo BRAMS para la región La Libertad-Perú}

\author{
Martha Nina (1) and Obidio Rubio(i)
}

Received, Mar. 12, 2020

Accepted, Jun. 10, 2020

How to cite this article:

Nina M, Rubio O. Validation of simulated precipitation forecasts with the BRAMS model for the La Libertad-Peru region. Selecciones Matemáticas. 2021;8(1):161-172. http://dx.doi.org/10.17268/sel.mat.2020. 01.15

\begin{abstract}
The objective of this work is to validate the precipitation forecasts simulated with the Brazilian Regional Atmospheric Modelling Systems (BRAMS) numerical model in its version 5.3, for the La Libertad Region in Peru; this region of Peru has $80 \%$ of its land located in the northern highlands of Peru. In mountainous areas, precipitation is strongly influenced by the height of the terrain, a poor representation of the topographic elevations and depressions of the terrain can lead to an erroneous representation of the resolvable phenomena explicit by the model. Taking into account this characteristic of the study area, in the first instance the BRAMS model was configured to adequately represent the rugged orography of the local area at a horizontal resolution of $10 \mathrm{~km}$. Then, the model was run to simulate precipitation forecasts at time horizons of 24, 48, 72, 96 and 120 hours, for the months of December 2019, January and February 2020. The validation of the forecasts was performed against observed data obtained from the National Meteorological and Hydrological Service (SENAMHI) of Peru, using quality indices for continuous and binary variables. From the results obtained, it is concluded that the BRAMS model performed well in forecasting the occurrence of precipitation for all time horizons. However, the model had difficulties in forecasting the occurrence of precipitation for higher thresholds, and predicted more false alarms for these thresholds. Finally, the model applied to the La Libertad Region in Peru, with a fairly rugged topography, had similar results to those obtained by other regional models applied in areas where there is little influence of terrain height.
\end{abstract}

Keywords . Numerical model BRAMS, quality evaluation, forecast verification, precipitation.

\section{Resumen}

El objetivo del presente trabajo es validar los pronósticos de precipitación simulados con el modelo numérico BRAMS (Brazilian Regional Atmospheric Modelling Systems), en su versión 5.3, para la Región de La Libertad en Perú; esta región del Perú tiene el $80 \%$ de terreno ubicado en la sierra norte del Perú. En zonas montañosas, la precipitación está fuertemente influenciada por la altura del terreno, una representación deficiente de las elevaciones y depresiones topográficas del terreno pueden conllevar a una representación errónea de los fenómenos resolubles explícitos por el modelo. Teniendo en cuenta esta característica de la zona de estudio, en primera instancia el modelo BRAMS fue configurado para representar adecuadamente la orografía accidentada del área local a una resolución horizontal de $10 \mathrm{~km}$. Luego, se ejecutó el modelo para simular pronósticos de precipitación a horizontes de tiempo de 24, 48, 72, 96 y 120 horas, para los meses diciembre de 2019, enero y febrero del año 2020. La validación de los pronósticos se ha

\footnotetext{
*Departamento de Matemática y Física, Universidad Nacional de San Cristóbal de Huamanga, Ayacucho, Perú. (martha.nina@unsch.edu.pe).

${ }^{\dagger}$ Departamento de Matemáticas, Universidad Nacional de Trujillo, Trujillo, Perú. (orubio@unitru.edu.pe).
} 
realizado frente a los datos observados obtenidos del Servicio Nacional de Meteorología e Hidrología (SENAMHI) del Perú, usando indicadores de calidad de pronósticos determinísticos continuos y binarias. De los resultados obtenidos, se concluye que el modelo BRAMS tuvo un buen desempeño para pronosticar la ocurrencia de precipitaciones para todos los horizontes de tiempo. Sin embargo, el modelo tuvo dificultades para pronosticar la ocurrencia de precipitaciones de umbrales mayores, y pronosticó mas cantidad de falsas alarmas para estos umbrales. Finalmente, el modelo aplicado a la Región de La Libertad en Perú con una topografía bastante accidentada, tuvo similares resultados a los obtenidos por otros modelos regionales aplicado en zonas donde hay poca influencia de la altura del terreno.

Palabras clave. Modelo numérico BRAMS; evaluación de calidad; verificación de pronósticos, precipitación.

1. Introducción. Los modelos numéricos de predicción computacional del tiempo son una herramienta muy importante, que hoy en día se utilizan para la predecir el tiempo en los servicios meteorológicos [2]. Los modelos numéricos regionales han evolucionado en los últimos años, actualmente se ejecutan con resolución horizontal de decenas de kilómetros, hasta metros, lo que permite una mejor representación de los fenómenos de mesoescala en una región dada del planeta. Estos modelos incorporan características regionales como la topografía, vegetación, tipo de suelo, etc. [3, 6, 16]

El modelo numérico BRAMS(Brazilian Regional Atmospheric Modelling Systems) en su versión 5.3 es un modelo regional de gran precisión, con una resolución cuatro veces superior a la versión anterior que sólo garantizaba una resolución de 20 kilómetros, es capaz de realizar previsiones hasta por siete días [3]. En países como Brasil y Argentina este modelo es ampliamente usado para generar pronósticos en tiempo real y también para realizar trabajos de investigación [4]. Usando le versión 4.2 del BRAMS, se han realizado simulaciones para el norte del Perú describiendo las variables temperatura, humedad relativa en [10] .

La validación de los pronósticos que son generados por un modelo numérico es esencial, porque nos permite conocer bajo qué circunstancias estos son confiables y que aspectos físicos, matemáticos y numéricos se debe mejorar; el propósito es ir mejorando dichas falencias.

Entre las variables meteorológicas, la precipitación es una de las más importantes a pronosticar, debido a que diversas actividades del hombre están condicionadas a la presencia de ellas. Los pronósticos de precipitación extrema, pueden ayudar a la población a prevenir situaciones de riesgo, como inundaciones, los cuales causan daño a la agricultura, a los ríos, etc. La precipitación es el agua procedente de la atmósfera y que en forma de llovizna, lluvia, nieve, aguanieve o granizo, se deposita sobre la superficie de la tierra. La precipitación se mide en milímetros de agua, es decir, se mide la altura en milímetros ( $\mathrm{mm}$ ) que alcanzaría la lámina de agua en una superficie horizontal de $1 \mathrm{~m}^{2}$ si el agua no se escurriera.

La precipitación es una de las variables difíciles de representar por los modelos numéricos, sobre todo en zonas donde la orografía es bastante compleja. Los efectos de una inadecuada representación del terreno pueden conllevar a una representación errónea de los fenómenos resolubles explícitamente por el modelo, y por tanto de las precipitaciones. Se han hecho algunos estudios sobre la precipitación usando una resolución anidada hasta $20 \mathrm{~km}$ con BRAMS 4.5 [11], para precipitaciones medias mensuales.

La región de La Libertad está ubicada en el noroeste del Perú, cuya capital es la ciudad de Trujillo, es el único departamento del Perú que abarca las 3 regiones naturales, Costa, Sierra y Selva, y salida al mar. Se caracteriza por tener una orografía altamente diversa, presenta una altitud que oscila entre los 3 y los 4 008 m.s.n.m. La costa está constituida por planicies fértiles y desérticas, la sierra por quebradas abruptas, valles y nevados originados por la existencia de la Cordillera de los Andes. La sierra a partir de los 3.000 m.s.n.m., tiene un clima seco y templado durante el día y frío en la noche, durante los meses de enero a marzo es un periodo de intensas lluvias en esta zona. El $80 \%$ de su área superficial es esencialmente sierra.

El objetivo del presente trabajo es validar los pronósticos de precipitación generados mediante simulaciones con el modelo BRAMS en su versión 5.3, para la región de La Libertad en Perú. Se ejecutó el modelo a $10 \mathrm{~km}$ de resolución horizontal para simular pronósticos de precipitación a horizontes de tiempo 24, 48, 72, 96 y 120 horas, desde diciembre-2019 hasta marzo-2020. La validación de los pronósticos se ha realizado frente a los datos observados obtenidos del Servicio Nacional de Meteorología e Hidrología (SENAMHI) del Perú, usando indicadores de calidad y gráficos. Para realizar las simulaciones se ha implementado el modelo BRAMS en el Centro de Cálculo del Departamento de Matemática de la Universidad Nacional de Trujillo. Para las visualizaciones gráficas y tablas se utilizó los softwares Grads y R.

\section{Modelo matemático.}

2.1. Ecuaciones del modelo. El modelo BRAMS resuelve numéricamente las ecuaciones no-hidrostáticas compresibles descritas en Tripoli y Cotton [16]. Todas estas ecuaciones son promedios de Reynolds, ellas son:

Ecuaciones del movimiento.

$\frac{\partial u}{\partial t}=-u \frac{\partial u}{\partial x}-v \frac{\partial u}{\partial y}-w \frac{\partial u}{\partial z}-\theta \frac{\partial \pi^{\prime}}{\partial x}+f v+\frac{\partial}{\partial x}\left(K_{m} \frac{\partial u}{\partial x}\right)+\frac{\partial}{\partial y}\left(K_{m} \frac{\partial u}{\partial y}\right)+\frac{\partial}{\partial z}\left(K_{m} \frac{\partial u}{\partial z}\right)$ 


$$
\begin{gathered}
\frac{\partial v}{\partial t}=-u \frac{\partial v}{\partial x}-v \frac{\partial v}{\partial y}-w \frac{\partial v}{\partial z}-\theta \frac{\partial \pi^{\prime}}{\partial y}-f u+\frac{\partial}{\partial x}\left(K_{m} \frac{\partial v}{\partial x}\right)+\frac{\partial}{\partial y}\left(K_{m} \frac{\partial v}{\partial y}\right)+\frac{\partial}{\partial z}\left(K_{m} \frac{\partial v}{\partial z}\right) \\
\frac{\partial w}{\partial t}=-u \frac{\partial w}{\partial x}-v \frac{\partial w}{\partial y}-w \frac{\partial w}{\partial z}-\theta \frac{\partial \pi^{\prime}}{\partial z}-\frac{g \theta_{v}^{\prime}}{\theta_{0}}+\frac{\partial}{\partial x}\left(K_{m} \frac{\partial w}{\partial x}\right)+\frac{\partial}{\partial y}\left(K_{m} \frac{\partial w}{\partial y}\right)+\frac{\partial}{\partial z}\left(K_{m} \frac{\partial w}{\partial z}\right)
\end{gathered}
$$

Ecuación de termodinámica.

$$
\begin{aligned}
\frac{\partial \theta_{i l}}{\partial t}=-u \frac{\partial \theta_{i l}}{\partial x}-v \frac{\partial \theta_{i l}}{\partial y}-w \frac{\partial \theta_{i l}}{\partial z}+ & \frac{\partial}{\partial x}\left(K_{h} \frac{\partial \theta_{i l}}{\partial x}\right)+\frac{\partial}{\partial y}\left(K_{h} \frac{\partial \theta_{i l}}{\partial y}\right)+\frac{\partial}{\partial z}\left(K_{h} \frac{\partial \theta_{i l}}{\partial z}\right)+\left(\frac{\partial \theta_{i l}}{\partial t}\right)_{r a d} \\
& +\left(\frac{\partial \theta_{i l}}{\partial t}\right)_{m i c}+\left(\frac{\partial \theta_{i l}}{\partial t}\right)_{c o n}
\end{aligned}
$$

Ecuación de continuidad de la relación de mezcla de especies de agua.

$$
\begin{gathered}
\frac{\partial r_{n}}{\partial t}=-u \frac{\partial r_{n}}{\partial x}-v \frac{\partial r_{n}}{\partial y}-w \frac{\partial r_{n}}{\partial z}+\frac{\partial}{\partial x}\left(K_{h} \frac{\partial w}{\partial x}\right)+\frac{\partial}{\partial y}\left(K_{h} \frac{\partial r_{n}}{\partial y}\right)+\frac{\partial}{\partial z}\left(K_{h} \frac{\partial r_{n}}{\partial z}\right) \\
+\left(\frac{\partial r_{v}}{\partial t}\right)_{m i c}+\left(\frac{\partial r_{T}}{\partial t}\right)_{c o n}
\end{gathered}
$$

Ecuación de continuidad para masa.

$$
\frac{\partial \pi^{\prime}}{\partial t}=-\frac{R \pi_{0}}{c_{v} \rho_{0} \theta_{0}}\left(\frac{\partial \rho_{0} \theta_{0} u}{\partial x}+\frac{\partial \rho_{0} \theta_{0} v}{\partial y}+\frac{\partial \rho_{0} \theta_{0} w}{\partial z}\right)
$$

2.2. Dominio del modelo. El dominio horizontal del modelo corresponde al área que abarca desde $-10,65^{\circ}$ a $-5,25^{\circ}$ de latitud y desde $-80,65^{\circ}$ a $-75,25^{\circ}$ de longitud. Este dominio cubre todo el departamento de La Libertad en Perú (ver figura 2.1).

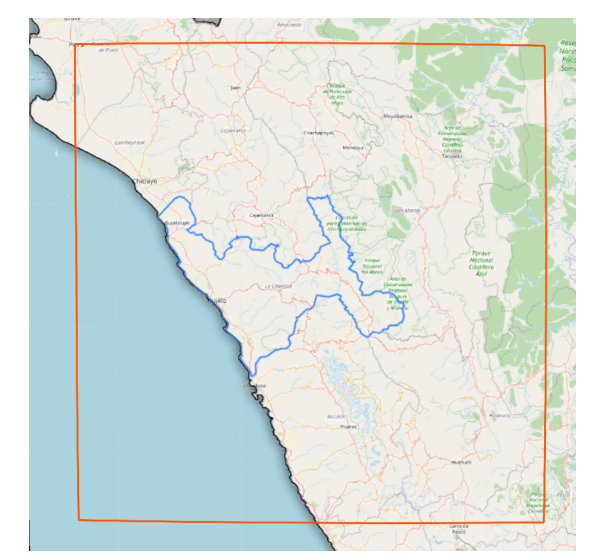

Figura 2.1: Dominio horizontal del modelo encerrado con línea roja

2.3. Variables y parámetros. En la Tabla 2.1 se muestra la lista de símbolos presentes en el modelo.

\begin{tabular}{|l|l|}
\hline Símbolo & Definición \\
\hline$u$ & Componente de viento de este-oeste \\
$v$ & Componente de viento de norte-sur \\
$w$ & Componente de viento vertical \\
$f$ & Parámetro Coriolis \\
$K_{m}$ & Coeficiente viscosidad turbulenta para momento \\
\hline
\end{tabular}




\begin{tabular}{|l|l|}
$K_{h}$ & Coeficiente viscosidad turbulenta de calor y vapor de agua \\
$\theta_{i l}$ & Temperatura potencial de agua hielo-líquido \\
$r_{n}$ & Relación de mezcla de especies de agua de agua total, lluvia, \\
& cristales prístinos, agregados y nieve \\
$\rho_{0}$ & Estado de referencia para la densidad del aire \\
$c o n$ & Subíndice que denota la tendencia de la parametrización convectiva \\
$R$ & Constante de gas aire seco \\
$r a d$ & subíndice que denota la tendencia de la parametrización de la radiación \\
$m i c$ & Subíndice que denota la tendencia de la parametrización microfísica \\
$g$ & a escala resoluble \\
$r_{T}$ & Gravedad \\
$r_{v}$ & Relación de mezcla de agua total \\
$\pi_{0}$ & Relación de mezcla de vapor de agua \\
$\pi^{\prime}$ & Estado de referencia para la función Exner \\
$\theta_{v}$ & Perturbación función Exner \\
$\theta_{0}$ & Temperatura potencial virtual \\
$c_{v}$ & Estado de referencia para temperatura potencial \\
\hline
\end{tabular}

Tabla 2.1: Lista de variables y parámetros

3. Modelo numérico BRAMS. El modelo BRAMS se deriva del RAMS(Regional Atmospheric Modelling Systems), éste último desarrollado originalmente en la Universidad Estatal de Colorado en los Estados Unidos. BRAMS/RAMS son modelos numéricos de predicción meteorológica multipropósito diseñados para simular circulaciones atmosféricas regionales que abarcan desde ondas a escala planetaria hasta grandes remolinos de la capa límite planetaria.

El modelo BRAMS es adaptado y rediseñado por investigadores de la Universidad de São Paulo (USP), el "Instituto Nacional de Pesquisas Espaciáis" (INPE), la Universidad Federal de Campina Grande (UFCG) y de otros centros de investigación de Brasil; se diferencia de RAMS, puesto que cuenta con varias características nuevas principalmente para mejorar la representación numérica de los procesos físicos en regiones tropicales y subtropicales de América del Sur.

La versión 5.3 tiene un nuevo conjunto de parametrizaciones físicas de última generación y una mayor eficiencia de uso de memoria y computación paralela [3]. El modelo está integrado en un solo software que unifica las diferentes versiones anteriores para el tiempo, el clima, la química y el ciclo de carbono.

Es un modelo regional o de mesoescala, está equipado con un esquema de anidamiento unidireccional (one-way-nest) y este tipo de modelos generalmente son más robustos con respecto a los de múltiples direcciones o anidados.

Para resolver las ecuaciones el modelo utiliza el método de diferencias finitas, para esto la atmósfera es dividida en celdas tridimensionales. Para representar y calcular las cantidades físicas presentes en el modelo se utiliza las mallas de Arakawa tipo C; las variables atmosféricas se calculan para cada celda, el código está codificado en el lenguaje FORTRAN y es de código abierto y distribuído bajo licencia CC-GPL.

3.1. Datos de entrada. El modelo BRAMS para ser ejecutado necesita datos para las condiciones iniciales y de frontera, además de otros, estos datos se encuentran disponibles junto al código y que provienen de fuentes como las que se mencionan a continuación:

- Uso de suelo o cobertura vegetal: Datos con resolución de $1 \mathrm{~km}$ proveniente del Servicio Geológico de Estados Unidos (USGS) de la FAO/UNESCO de las Naciones Unidas. Estos datos contienen los tipos globales de textura del suelo.

- Índice de vegetación de diferencia normalizada de MODIS: Datos procesados por "Terrestrial Biophysics and Remote Sensing Lab"(http://tbrs.arizona.edu/cdrom/Index.html); este conjunto de datos global mensual tiene una resolución de espacio de 30 segundos. 
- Humedad del suelo: Datos que tienen una estructura espacial en ocho capas del suelo en términos de humedad. Se usó los datos de GPNR que utiliza la precipitación del programa "Tropical Rainfall Measuring Mission”(TRMM) de la NASA (http://trmm.gsfc.nasa. gov), cuya resolución es de $0,25 \times 0,25$ grados.

- Temperatura de la superficie de mar (SST): Datos provenientes del "Center for Environmental Prediction"(NCEP) de la "National Oceanic and Atmospheric Administration"(NOAA) en su versión OI.v2. datos semanal.

- Topografía: Datos de topografía global de la USGS de resolución 1 km.

- Estado atmosférico: Datos que contienen las condiciones iniciales y de frontera. Para las condiciones iniciales y de frontera se usó los datos de análisis atmosférico y los pronósticos del modelo “Global Forecast System"(GFS) de la NOAA (http://nomads.ncep.noaa.gov/pub/data/nccf/com/gfs/prod/), respectivamente. Estos datos tienen una resolución horizontal de $0,25 \times 0,25$ grados.

Estos datos a excepción de las condiciones iniciales y de frontera se encuentran disponibles en el Centro de Previsión de Tiempo y Estudios Climáticos (CPTEC) del Instituto Nacional de Investigaciones Espaciales de Brasil (INPE).

3.2. Configuración del modelo. El modelo fue configurado con una resolución horizontal de $10 \mathrm{~km} \mathrm{y}$ temporal de 1 hora, con 61 puntos de latitud, 61 puntos de longitud y 35 niveles en la vertical, alcanzando el tope aproximadamente de $19 \mathrm{~km}$ de altura. Se consideró el modelo no hidrostático y compresible, se utilizó la coordenada sigma- $Z$ de seguimiento del terreno para el sistema de coordenada vertical y la coordenada estereográfica polar transformada para el sistema de coordenada horizontal.

El modelo BRAMS se inicia con los datos de análisis del modelo global GFS y es forzado lateralmente para mantenerse cerca de los pronósticos del modelo global a lo largo del periodo de simulación mediante la asimilación de datos usando el método variacional de 4 dimensiones. Para la integración del tiempo se consideró el esquema numérico al filtro de tiempo de Robert-Asselin-William [18], y para la advección se utilizó el esquema forward upstreams de 2do orden no monotónico [15]. Para la radiación se usó el esquema de radiación de onda corta y onda larga RRTMG [5], para la microfísica la formulación de Greg Thomson de un momento [14] y para la turbulencia el esquema de Mellor y Yamada en la vertical y de Smagorinsky en la horizontal [9]. Se desactiva las parametrizaciones de la convección profunda como la superficial o poco profunda, el modelo trata la convección profunda de forma explícita [2].

La configuración del modelo se resume en la Tabla 3.1.

\begin{tabular}{|l|l|}
\hline Malla del dominio & \\
Resolución horizontal & $10 \mathrm{~km}$ \\
Número de nodos en dirección X e Y & 61 \\
Número de capas verticales & 35 \\
Tiempo de paso & $30 \mathrm{~s}$ \\
Tiempo máximo de simulación & $120 \mathrm{~h}$ \\
Sistema de coordenadas horizontales & Proyección polar estereográfica \\
Sistema de coordenadas verticales & Coordenada sigma-Z de seguimiento de terreno \\
Anidamiento & One-way \\
\hline Análisis de inicialización & \\
Asimilación de datos & Método variacional 4D-dimensional \\
\hline Dinámica: Métodos numéricos & \\
Esquema de integración del tiempo & Filtro de tiempo de Robert-Asselin-William (William, \\
Esquema de integración para advección & Forward upstreams de 2do orden no monotónico (Trem- \\
\hline Parametrizaciones físicas & back et al., 1987) \\
\hline Radiación de onda corta y larga & Momento de Greb. Thomson (Thomson et al., 2014) \\
\hline Microfísica de nubes & \\
\hline
\end{tabular}


Tabla 3.1: Configuración del modelo BRAMS

3.3. Ajuste en el módulo de topografía del modelo. La sierra del Departamento de La Libertad es una región que tiene una orografía variada originada por la existencia de la Cordillera de los Andes, podemos encontrar montañas, colinas, valles y zonas elevados, por lo que la circulación y flujo horizontal del aire en la atmósfera sobre la superficie terrestre de esta zona está sometido a obstáculos que limitan su flujo.

La altura del terreno tiene una influencia importante en muchos fenómenos meteorológicos, en particular, la precipitación está fuertemente influenciada por el terreno, principalmente en zonas montañosas [8]. Se debe tener en cuenta que el modelo simulará los fenómenos atmosféricos a partir de la información topográfica que se le suministre. Cuanto menos precisa sea ésta, los resultados de la modelización presentarán menor exactitud.

Los parámetros que fueron analizados en el módulo de topografía del archivo RAMSIN del modelo BRAMS son los siguientes.

- ITOPSFLG: Esta variable controla el método para procesar los datos de topografía ingresados, con el propósito de representar la altura del terreno en cada malla del modelo. Se tiene las siguientes opciones:

0 = Promedio orográfico,

1 = Promedio de silueta orográfica,

$2=$ Promedio de envolvente orográfica,

3 = Promedio de Envolvente Reflejada orográfica.

- TOPTENH: Esta variable depende de la elección de ITOPSFLG. Si ITOPSFLG=1, TOPTENH es el peso asignado al calcular el promedio ponderado entre el promedio orográfico y el promedio de la silueta asignado, y si ITOPSFLG=2 ó 3, TOPTENH corresponderá a un factor de mejora que multiplica la desviación estándar dentro de la formulación.

- TOPTWVL: Variable que especifica la longitud de onda, en unidades de tamaño de celda, de los modos (de propagación) horizontal más pequeño, de los datos de altura del terreno que están presentes en la malla del modelo dado.

\begin{tabular}{|c|c|c|c||c|c|c|c|}
\hline Simulación & ITOPSFLG & TOPTENH & TOPTWVL & Simulación & ITOPSFLG & TOPTENH & TOPTWVL \\
\hline sim-1 & 1 & 1 & 2 & sim-11 & 3 & 1.5 & 3 \\
sim-2 & 1 & 1 & 3 & sim-12 & 3 & 2 & 3 \\
sim-3 & 1 & 1 & 4 & sim-13 & 3 & 2.5 & 3 \\
sim-4 & 1 & 1 & 6 & sim-14 & 3 & 3 & 3 \\
sim-5 & 2 & 1 & 2 & sim-15 & 3 & 1 & 4 \\
sim-6 & 2 & 1 & 3 & sim-16 & 3 & 1 & 6 \\
sim-7 & 2 & 1 & 4 & sim-17 & 3 & 2 & 4 \\
sim-8 & 2 & 1 & 6 & sim-18 & 3 & 2 & 6 \\
sim-9 & 3 & 0.5 & 3 & sim-19 & 3 & 3 & 4 \\
sim-10 & 3 & 1 & 3 & sim-20 & 3 & 3 & 6 \\
\hline
\end{tabular}

Tabla 3.2: Simulaciones realizadas para el análisis de sensibilidad en el módulo de topografía

Las opciones consideradas para el estudio de sensibilidad de representación de la topografía en el modelo se muestran en la Tabla 3.2. Para el parámetro ITOPSFLG se consideró las opciones: Promedio de silueta, de envolvente y envolvente reflejada. Se ha mantenido constante la ponderación del esquema de silueta y factor de mejora para la envolvente orográfica (TOPTENH=1), y para la envoltura reflejada se realizó simulaciones con múltiples opciones en el parámetro TOPTENH, puesto que la zona de estudio tiene una orografía diversa, y este método tiene la bondad de preservar el efecto de barrera de la zonas altas, además de la profundidad de los valles.

Los datos de topografía utilizados para representar las altidudes en el modelo BRAMS, son los datos de elevación digital de la USGSS de los Estados Unidos, a $1 \mathrm{~km}$ de resolución. Para el análisis de sensibilidad se realizaron 20 simulaciones tal como se muestra en la Tabla 3.2.

4. Validación de pronosticos de la Variable Precipitación. La validación del pronóstico de la varaible simulada lo llevamos a cabo con los datos observados, para ello utilizamos los índices estadísticos, 
los que serán calculados en primer lugar para el ajuste de la topografía y luego para las simulaciones de la precipitación.

La validación de pronósticos es el proceso de evaluar la calidad (bondad) de los pronósticos. Este proceso puede ser cualitativo o cuantitativo; el objetivo es proporcionar información sobre la naturaleza de los errores del pronóstico [6]. Cualquier método de validación de pronósticos implica necesariamente comparación entre pronósticos y observaciones.

4.1. Datos de observaciones . Los datos observados de precipitación utilizados en este trabajo son recogidos de las Estaciones Meteorológicas del Servicio Nacional de Meteorología e Hidrología del Perú (SENAMHI). Las Estaciones fueron seleccionadas, de acuerdo a las recomendaciones de la Organización Mundial de Meteorología (OMM) y cumplen los siguientes criterios: están ubicadas dentro del dominio del modelo a una distancia mayor a $10 \mathrm{~km}$ una de la otra y situadas por encima de los 1000 m.s.n.m. En total son ocho Estaciones que cumplen estos criterios y se encuentran ubicadas en el Departamento de La Libertad. En la Tabla 4.1 se muestra las características de estas estaciones.

\begin{tabular}{|r|l|c|r|r|r|}
\hline & Estación & Tipo & m.s.n.m & Latitud & Longitud \\
\hline 1 & Cachicadán & $\mathrm{C}$ & 2900 & -8.091844 & -78.149383 \\
2 & Huamachuco & $\mathrm{C}$ & 3186 & -7.819158 & -78.040133 \\
3 & Salpo & $\mathrm{C}$ & 3418 & -8.005344 & -78.607103 \\
4 & Callancas & $\mathrm{C}$ & 1501 & -7.767500 & -78.477286 \\
5 & Mollepata & $\mathrm{C}$ & 2708 & -8.191369 & -77.953972 \\
6 & Quiruvilca & $\mathrm{C}$ & 4047 & -8.004222 & -78.307911 \\
7 & Huangacocha & $\mathrm{C}$ & 3763 & -7.937608 & -78.068108 \\
8 & Julcan & $\mathrm{C}$ & 3385 & -8.042286 & -78.486069 \\
\hline
\end{tabular}

Tabla 4.1: Estaciones meteorológicas consideradas para el estudio.- Fuente: SENAMHI. C: Convencional.

4.2. Índices de calidad para validación de pronósticos. La validación de los pronósticos de precipitación generados con el modelo BRAMS se realizó mediante los estadísticos o también denominados índices de calidad: bias (B), probabilidad de detección (probability of detection, POD), relación de falsa alarma (false alarm ratio, FAR) y puntuación de amaneza equitativa (equitable threat score, ETS) [6, 1, 17]. Estos estadísticos de definen como sigue:

$$
B=\frac{a+b}{a+c}, \quad P O D=\frac{a}{a+c}, \quad F A R=\frac{b}{a+b}, \quad E T S=\frac{a-a_{r}}{a+b+c-a_{r}},
$$

con

$$
a_{r}=\frac{(a+b)(a+c)}{N}
$$

donde $N=a+b+c+d$ es el total del tamaño de la muestra, $a$ es el número de eventos que se pronosticó y se observó (aciertos), $b$ número de eventos que se pronosticó y no se observó (falsas alarmas), $c$ número de eventos que no se pronosticó y se observó (fallas) y $d$ número de eventos que no se pronosticó y no se observó (negativos correctos).

- Bias o sesgo, mide la relación entre el número de eventos pronosticados y el número de eventos que se observó. Rango: $[0,+\infty\rangle$. Puntuación perfecta 1 .

- POD describe la relación entre el número de eventos que se pronosticó correctamente (aciertos) y el número de eventos que se observó o que ocurrieron. Rango: [0,1]. Puntuación perfecta 1.

- FAR mide la relación entre el número de falsas alarmas y el número de eventos que se pronosticó. Rango: [0,1]. Puntuación perfecta es 0.

- ETS es un índice de habilidad frente a una medida de referencia. En este caso, la medida de referencia indica la ocasión al azar o aleatorio. Rango: $[-1 / 3,1]$. Puntuación perfecta 1.

Además, de los estadísticos indicados, para complementar la evaluación de los pronósticos y para realizar el ajuste en el modulo de topografía del modelo se consideró el error medio (mean error, ME), error absoluto medio (mean absolute error, MAE), raíz del error cuadrático medio (root mean square error, 
RMSE), y el índice de correlación de Pearson $(r)$, los cuales se definen somo sigue:

$$
\begin{gathered}
M E=\frac{1}{N} \sum_{i=1}^{N}\left(P_{i}-O_{i}\right), \quad M A E=\frac{1}{N} \sum_{i=1}^{N}\left|P_{i}-O_{i}\right|, \quad R M S E=\sqrt{\frac{1}{N} \sum_{i=1}^{N}\left(P_{i}-O_{i}\right)^{2}} \\
r=\left(\sum_{i=1}^{N} \frac{\left(P_{i}-\bar{P}\right)\left(O_{i}-\bar{O}\right)}{\sqrt{\sum_{i=1}^{N}\left(P_{i}-\bar{P}\right)^{2}} \sqrt{\sum_{i=1}^{N}\left(O_{i}-\bar{O}\right)^{2}}}\right)
\end{gathered}
$$

donde $N$ es el número total de observaciones o tamaño de muestra, $P_{i}$ es valor pronosticado, $O_{i}$ es el valor observado, $\bar{P}$ y $\bar{O}$ es el promedio de los pronósticos y observaciones, respectivamente.

El rango de ME es $\mathbb{R}$, de MAE y RMSE es $[0, \infty\rangle$, y de $r$ es $[-1,1]$. Mientras que la puntuación perfecta para ME, MAE y RMSE es 0 y para $r$ es 1 .

4.3. Validacion de la topografia. Para realizar las validaciones de las altitudes representadas por el modelo frente a las altitudes de las ocho Estaciones Meteorológicas consideradas, se utilizó los indicadores como el Error medio (ME), Error Absoluto Medio (MAE), Raíz del Error Cuadrático Medio (RMSE) y el Coeficiente de correlación estadística o coeficiente de correlación de Pearson $(r)$. En la Tabla 4.2 se muestra los resultados obtenidos.

\begin{tabular}{|c|c|c|c|c|c|c|c|c|c|}
\hline Simulación & ME & MAE & RMSE & r & Simulación & ME & MAE & RMSE & r \\
\hline sim-1 & -359 & 389 & 538 & 0.747 & sim-11 & -133 & 226 & 267 & 0.925 \\
\hline sim-2 & -487 & 516 & 668 & 0.6705 & sim-12 & -132 & 218 & 253 & 0.9293 \\
\hline sim-3 & -579 & 614 & 785 & 0.422 & sim-13 & -130 & 218 & 252 & 0.9307 \\
\hline sim-4 & -701 & 748 & 931 & 0.032 & sim-14 & -104 & 195 & 225 & 0.9426 \\
\hline sim-5 & -338 & 371 & 516 & 0.7569 & sim-15 & -252 & 305 & 454 & 0.7649 \\
\hline sim-6 & -438 & 456 & 601 & 0.7769 & sim-16 & -358 & 521 & 694 & 0.2175 \\
\hline sim-7 & -545 & 561 & 720 & 0.5989 & sim-17 & -347 & 356 & 520 & 0.7842 \\
\hline sim-8 & -668 & 720 & 894 & 0.1277 & sim-18 & -590 & 650 & 835 & 0.2423 \\
\hline sim-9 & -136 & 251 & 323 & 0.8991 & sim-19 & -443 & 471 & 623 & 0.7915 \\
\hline sim-10 & -135 & 238 & 291 & 0.916 & sim-20 & -821 & 826 & 1016 & 0.2494 \\
\hline
\end{tabular}

Tabla 4.2: Resultados del análisis de sensibilidad en el módulo de topografía

De los resultados del análisis de sensibilidad en la topográfia, la simulación sim-14 presenta menor error ME, MAE y RMSE, esto significa que para la configuración correspondiente las alturas aproximadas presentan mayor exactitud. Además, la simulación sim-14 presenta el mayor valor del índice de correlación de Pearson $r=0,9426$ lo que indica que las alturas reales con los aproximados están correlacionados

\begin{tabular}{|c|c|c|c|c|c|c|c|c|c|c|c|c|c|c|c|c|c|c|c|c|}
\hline & \multicolumn{20}{|c|}{ Simulaciones $(\operatorname{Sim}=x)$} \\
\hline & $\mathbf{x}=1$ & 2 & 3 & 4 & 5 & 6 & 7 & 8 & 9 & 10 & 11 & 12 & 13 & 14 & 15 & 16 & 17 & 18 & 19 & 20 \\
\hline 1 & 20 & 26 & 26 & 35 & 19 & 24 & 23 & 31 & 12 & 11 & 10 & 8 & 7 & 5 & 12 & 21 & 10 & 27 & 9 & 33 \\
\hline 2 & 9 & 11 & 18 & 23 & 9 & 9 & 16 & 21 & 2 & 4 & 6 & 8 & 10 & 11 & 9 & 12 & 12 & 17 & 15 & 21 \\
\hline 3 & 2 & 4 & 8 & 9 & 3 & 2 & 9 & 10 & 4 & 2 & 1 & 1 & 2 & 3 & 3 & 3 & 11 & 8 & 19 & 19 \\
\hline 4 & 38 & 55 & 62 & 74 & 34 & 51 & 51 & 71 & 22 & 19 & 16 & 13 & 10 & 7 & 12 & 48 & 3 & 57 & 5 & 66 \\
\hline 5 & 41 & 44 & 55 & 61 & 41 & 39 & 54 & 60 & 23 & 21 & 19 & 17 & 14 & 12 & 43 & 54 & 48 & 64 & 54 & 75 \\
\hline 6 & 2 & 3 & 3 & 5 & 1 & 2 & 2 & 5 & 7 & 5 & 4 & 3 & 1 & 0 & 5 & 11 & 1 & 6 & 3 & 0 \\
\hline 7 & 4 & 7 & 6 & 5 & 4 & 7 & 6 & 4 & 3 & 5 & 7 & 9 & 11 & 7 & 2 & 2 & 6 & 2 & 11 & 7 \\
\hline 8 & 2 & 5 & 6 & 11 & 2 & 5 & 6 & 12 & 0 & 1 & 2 & 3 & 4 & 5 & 1 & 2 & 5 & 11 & 9 & 21 \\
\hline
\end{tabular}
linealmente.

Tabla 4.3: Error relativo porcentual de la altitud aproximada por el modelo BRAMS

En la Tabla 4.3, para cada simulación realizada, se muestra el error relativo porcentual de las altitudes representadas por el modelo frente a la altitud de cada Estación Meteorológica. Se observa que la simulación sim-14, presenta errores relativos entre 0 y $12 \%$, mientras que para el resto este intervalo es mayor. 
En conclusión, la configuración de la simulación sim-14 presenta mayor exactitud al representar la altitud de las Estaciones, con un error porcentual que no supera el $12 \%$. Esta simulación corresponde al uso del método de promedio de envolvente reflejada orográfica con un factor de mejora igual a 3 y con longitud de ondas de propagación aproximado de $30 \mathrm{~km}$. Los resultados de esta etapa serán considerados en la configuración global del modelo BRAMS.

4.4. Simulación numérica. El modelo BRAMS se ejecutó con la configuración segun se indica en la Tabla 3.1, junto al resultado del análisis de sensibilidad en el módulo de topografía. El modelo es inicializado a las 00 UTC, a 120 horas de pronósticos, durante 90 días que corresponden a los meses de diciembre del año 2019, enero y febrero del año 2020.

4.5. Método de validación de los pronósticos de precipitación. Los datos de precipitaciones observados provienen de las ocho Estaciones consideradas en la Tabla 3.1, proporcionados por el SENAMHI Perú, para los días que corresponden a los meses de diciembre de 2019, enero y febrero del 2020. Cada dato representa precipitación acumulada a 24 horas, medida en milímetros ( $\mathrm{mm})$.

Del conjunto de los datos observados se determinó los umbrales de precipitación: $u=0.1,8.6,14.64$, 17.95 y $25.44 \mathrm{~mm} /$ día; los últimos cuatros valores de $u$ corresponden a los percentiles 75, 90, 95 y 99 de todos los datos observados, provehidos por SENAMHI así mismo el primer umbral.

Se entenderá por evento la ocurrencia de precipitación, en un dia, mayor a un umbral $u$ indicado. Cuando la precipitación es mayor al umbral $0.1 \mathrm{~mm} /$ día indicará la ocurrencia de precipitación; mientras cuando la precipitación es mayor al umbral $25.44 \mathrm{~mm} /$ día indicará la ocurrencia de precipitaciones intensas o extremas.

Los pronósticos de precipitación simulados con el modelo BRAMS son generados para diferentes horizontes de tiempo: 24, 48, 72, 96 y 120 horas; es decir, los pronósticos son hasta por 5 días. Los pronósticos considerados para la validación corresponden a los puntos de ubicación de cada Estación considerada, para los días de los meses de diciembre-2019, enero y febrero del 2020.

La validación de los pronósticos de precipitación frente a sus observados se realizó considerando la precipitación como variable binaria (sí o no sucedió el evento). Para pronósticos de la precipitación como variable binaria se utilizó los estadísticos B, POD, FAR y TS; mientras que se consideró la precipitación como variable continua para medir el error ME, MEA, RMSE y el coeficiente de correlación de Pearson $r$.

La validación de los pronósticos de precipitación se ha realizado para cada horizonte de tiempo (24, $48,72,96$ y 120 horas) y para cada umbral considerado, obteniéndose diferentes valores de los estadísticos en cada caso.

5. Resultados y discusión. En las figuras 5.1, 5.2, 5.3 y 5.4 se muestran los valores de los estadísticos POD, FAR, ETS y BIAS, respectivamente, para los distintos umbrales de precipitación y diferentes horizontes de tiempo, determinados para precipitaciones acumuladas a 24 horas con el modelo BRAMS versión 5.3 a 10 km de resolución.

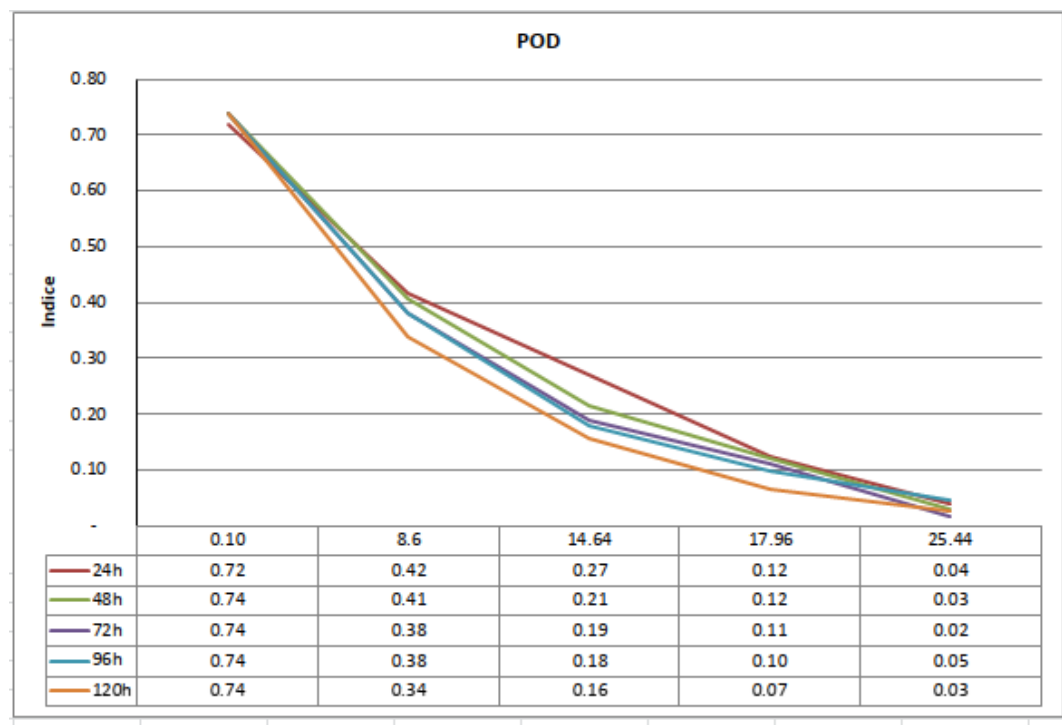

Figura 5.1: Probabilidad de acierto (POD) para distintos horizontes de tiempo. $x$ : Umbral, $y$ : Índice

En la figura 5.1, se observa que los valores de POD oscilan entre 0.72 y 0.74 para el umbral de 0.1 $\mathrm{mm}$, mientras que para el umbral $8.6 \mathrm{~mm}$ este valor se encuentra entre 0.34 y 0.42 , y para los demás 
umbrales estos valores están por debajo de 0.27. Sin embargo, en el trabajo de [7] los valores de POD determinados son inferiores a 0.2 en umbrales por debajo de $1 \mathrm{~mm}$ y en el trabajo de [13] estos resultados son muy similares. Por consiguiente, los valores que se obtuvo es este trabajo se encuentran por encima de los hallados en los trabajos antes mencionados, alcanzando su máximo valor de 0.74 para el umbral 0.1 $\mathrm{mm}$ y de 0.42 para el umbral $8.6 \mathrm{~mm}$. Asimismo, los valores de POD decrecen a medida que aumenta el umbral de precipitación, lo que indica que el modelo tiene dificultades para representar la ocurrencia de precipitaciones de umbrales mayores.

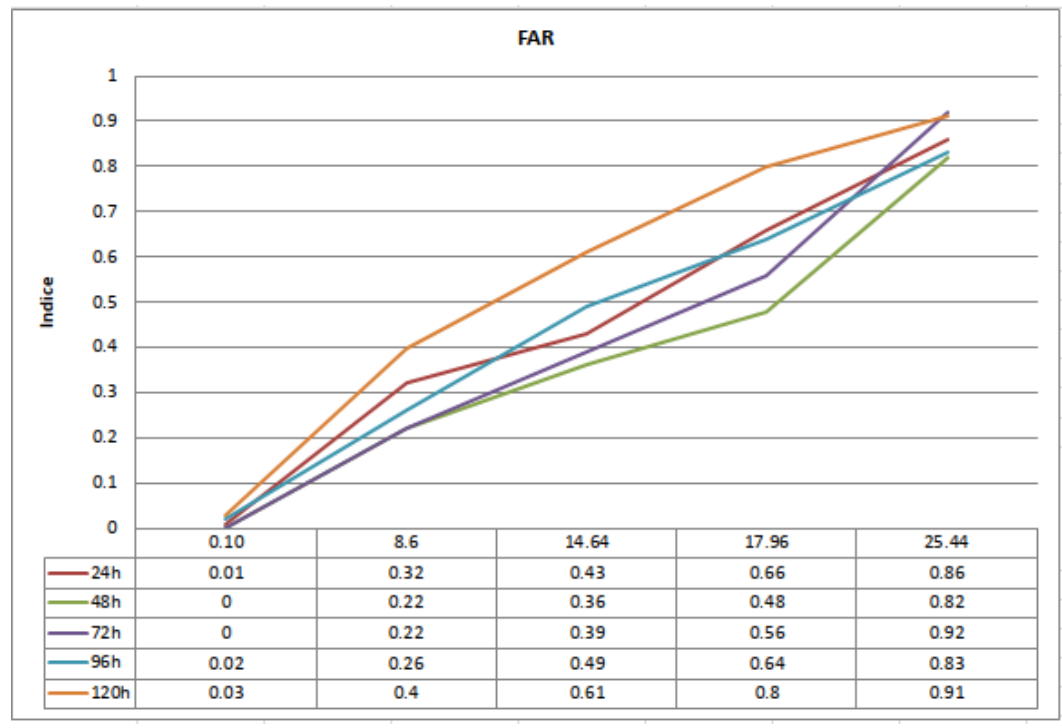

Figura 5.2: Tasa de falsa alarma (FAR) para distintos horizontes de tiempo. $x$ : Umbral, $y$ : Índice

En contraparte a POD (ver Figura 5.2), el índice FAR oscila entre 0 y 0.03 para el umbral $0.1 \mathrm{~mm}$, mientras que para el umbral $8.6 \mathrm{~mm}$ se encuentra entre 0.22 y 0.4 y para los umbrales mayores estos valores son superiores a 0.40 , llegando como máximo a 0.92. Siendo el valor perfecto de FAR igual a cero, los valores obtenidos son muy grandes a excepción para el umbral $0.1 \mathrm{~mm}$. Sin embargo, en los trabajos de [7] y [13], estos valores fueron inferiores a 0.4 para umbrales bajos, los cuales son muy similares a los que se obtuvo. Además, a medida que el umbral crece, este índice crece alcanzado su máximo de 0.92 para el umbral de $25.44 \mathrm{~mm}$, lo que indica que el modelo pronostica con mas frecuencia falsas alarmas a medida que el umbral y el horizonte de tiempo crecen.

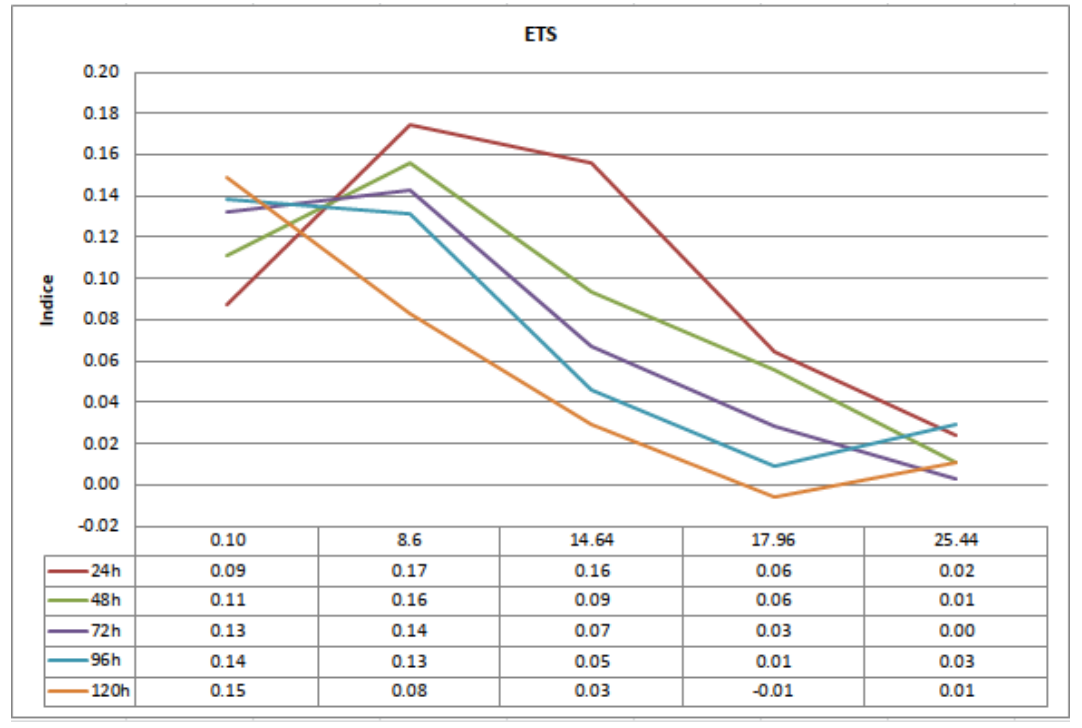

Figura 5.3: Puntuación de amenaza (TS) para distintos horizontes de tiempo. $x$ : Umbral, $y$ : Índice

De los valores mostrados en la figura 5.3, se observa que el valor máximo alcanzado por ETS es 0.17 para el umbral $8.6 \mathrm{~mm}$; mientras que los demás valores son inferiores a $0.17 \mathrm{~mm}$ llegando a un mínimo 
de cero. Se sabe que el valor ideal de este índice es 1 y los valores obtenidos están muy alejados de este. Sin embargo, en los trabajos de [2] y otros citados en este, encontramos valores de hasta 0.3 para eventos de precipitación acumulada a 12 horas con el modelo WRF-ARW (Advanced Research WRF) de $8 \mathrm{~km}$ de resolución, y en el trabajo de [7] estos valores alcanzaron a 0.2 para umbrales de $1 \mathrm{~mm}$ con el modelo BRAMS a 2 y $8 \mathrm{~km}$ de resolución. Esto indica que los valores que se obtuvo con el modelo BRAMS son muy próximos a los obtenidos por [7].

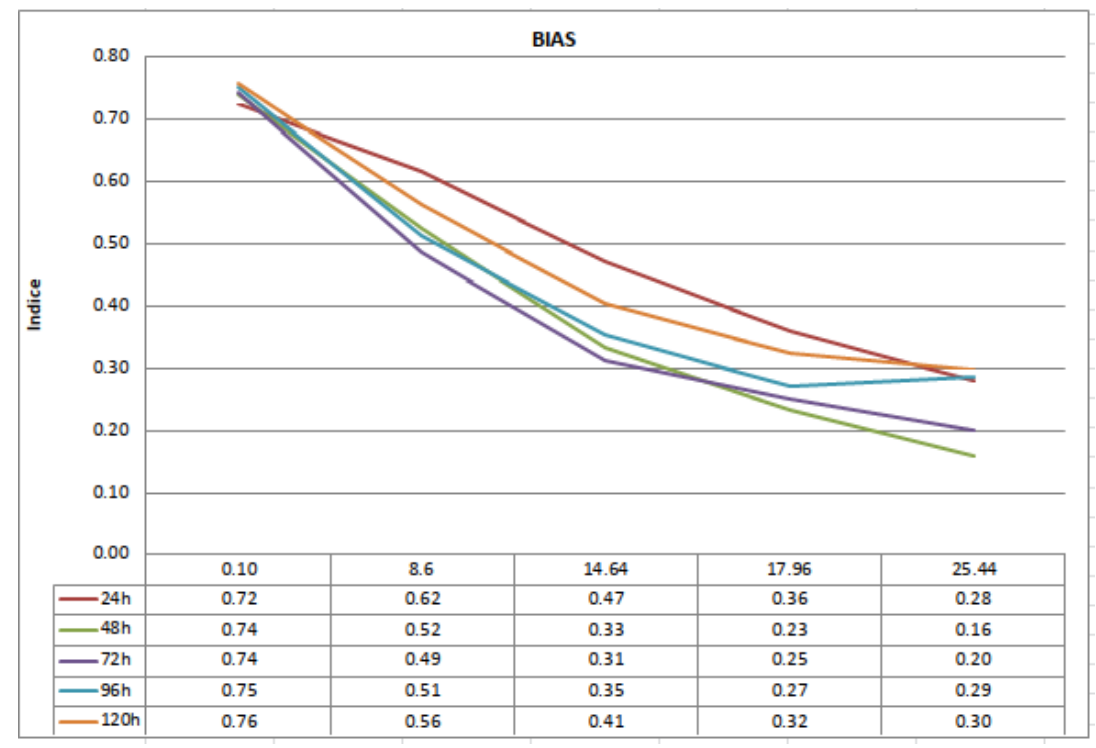

Figura 5.4: BIAS (B) para distintos horizontes de tiempo. $x$ : Umbral, $y$ : Índice

De acuerdo a la figura 5.4, se observa que los valores de Bias son menores que 1, lo que indica que el modelo subestimó las precipitaciones, sobretodo en zonas con precipitaciones de umbrales mayores a 14.64 mm que muestran valores de Bias por debajo de 0.36. Además, vemos que cuando el umbral crece, BIAS se aleja cada vez del valor perfecto que es 1. En el trabajo de [7] el modelo tiende a subestimar, siendo el umbral de $20 \mathrm{~mm}$ en el que más se aleja del valor perfecto, y en el trabajo de [2], el modelo sobrestimó en áreas con precipitaciones en umbrales bajos y en mayores a $25 \mathrm{~mm}$. De acuerdo a los valores que se observa para el umbral $0.1 \mathrm{~mm}$, Bias muestra sus mejores valores que oscilan entre 0.72 y 0.76 .
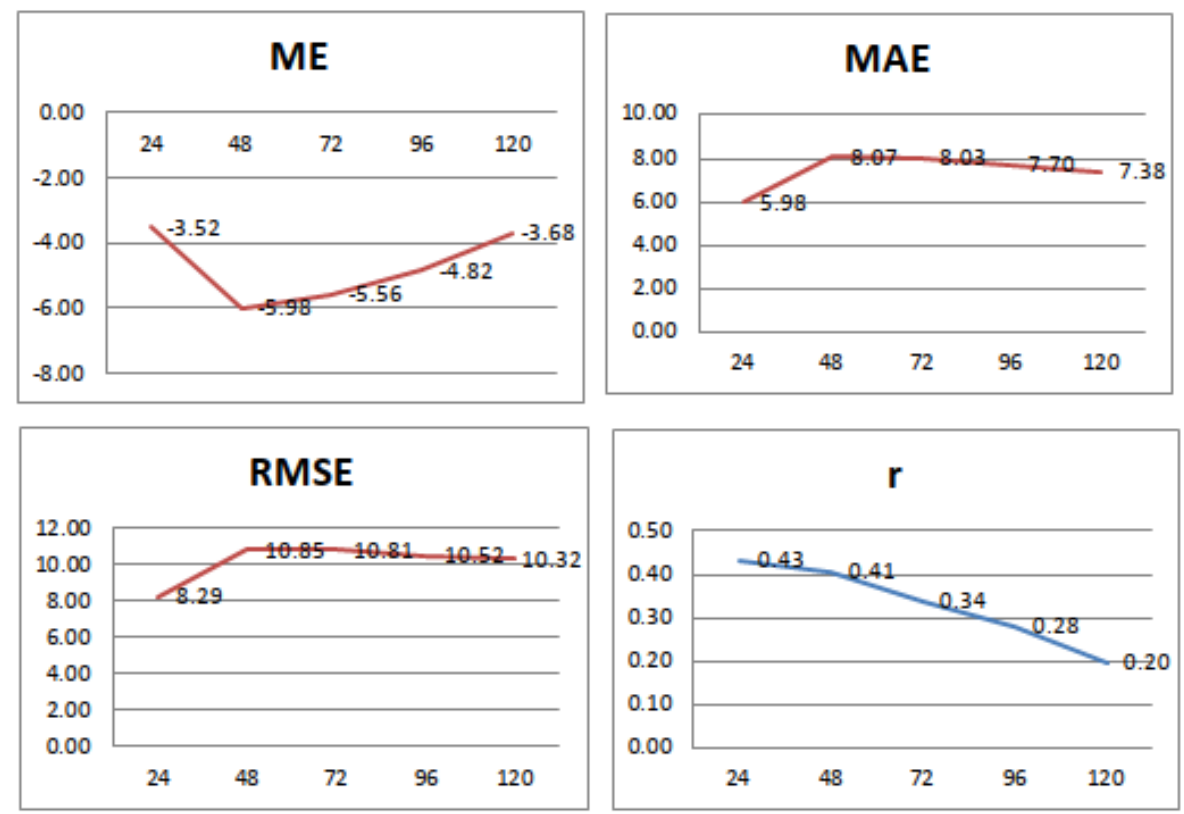

Figura 5.5: Error Medio (ME), Error Absoluto Medio (MAE), Raíz Cuadrada de Error Cuadrático Medio (RMSE), Coeficiente de Correlación de Pearson $(r)$ calculados para cada horizonte de tiempo 
En la Figura 5.5, los mínimos valores de MAE y RMSE son alcanzados para los pronósticos a 24 horas, asimismo MAE es el valor más cercano a cero para este horizonte de tiempo. Todos los valores de ME son negativos, lo que indica que las precipitaciones pronosticadas son subestimadas. También se observa que conforme pasa el tiempo estos errores crecen. Finalmente, el valor máximo de la correlación de Pearson es $r=0,43$ que se obtiene para pronósticos a 24 horas, esto significa que la correlación lineal de las precipitaciones observadas y pronosticadas es moderada [12].

6. Conclusiones. En este trabajo se ha realizado la validación de los pronósticos de precipitación frente a los observados para horizontes de tiempo de 24, 48, 72, 96 y 120 horas, para los días que corresponden a los meses de diciembre del año 2019, enero y febrero del año 2020, para un dominio que cubre la región de La Libertad en Perú con resolución horizontal de $10 \mathrm{~km}$. Para esto se ha determinado los valores de los estadísticos POD, FAR, ETS y Bias para los umbrales 0.1, 8.6, 14.64, 17.95 y 25.44 mm y para todos los horizontes de tiempo antes mencionados. Adicionalmente, se ha determinado los errores ME, MAE, RMSE y el índice de correlación de Pearson $r$,

De acuerdo a los resultados obtenidos, se concluye que el modelo BRAMS tuvo un buen desempeño para pronosticar la ocurrencia de precipitaciones para todos los horizontes de tiempo. Sin embargo, el modelo tuvo dificultades para pronosticar la ocurrencia de precipitaciones de umbrales mayores para todos los horizontes de tiempo, y pronosticó más cantidad de falsas alarmas para estos umbrales.

Finalmente, el modelo BRAMS en su versión 5.3, aplicado a la Región de La Libertad en Perú con una topografía bastante accidentada, tuvo similares resultados a los obtenidos por otros modelos regionales como el WRF y el propio BRAMS en sus versiones anteriores aplicado en zonas más llanas donde hay poca influencia de la altura del terreno. Siendo este modelo más eficiente que su versión anterior, se tiene la necesidad de seguir mejorando en la configuración del terreno para zonas montañosas, así como de disminuir la resolución horizontal con el objetivo de mejorar los pronósticos para umbrales mayores.

ORCID and License

Martha Nina https://orcid.org/0000-0002-2015-4512

Obidio Rubio https://orcid.org/0000-0002-6853-8160

This work is licensed under the Creative Commons - Attribution 4.0 International (CC BY 4.0)

\section{Referencias}

[1] Coello C,Brow B, Wilson L, Mittemaier B, Casati B. Forecats verification for S2S timescales. Elsevier Inc. 2019; 29 (1), 11-21.

[2] Dillon M, Skabar Y, Nicolini M. Desempeño del pronóstico de modelos de alta resolución, en un área limitada: análisis de la estación de verano 2010-2011. Meteorológica. 2013; 38:69-89.

[3] Freitas S, Panetta J, Longo K, Rodrigues L, Moreira D, Rosario N, y otros. The Brazilian developments on the Regional Atmospheric Modeling. Geosci. Model Dev. 2017; 189-222.

[4] García Y, Salio P, Nicolini M. Verificación de los pronósticos del modelo BRAMS centrado en la región subtropical de Sudámerica. Meteorología. 2012; 27(3).

[5] Iacono MJ, Delamere JS, Mlawer EJ, Shephard MW, Clough SA and Collins WD. Radiative forcing by long-lived greenhouse gases: Calculations with the AER radiative transfer models. J. Geophys. Res. 2008; 113:D13103. doi:10.1029/2008JD009944.

[6] Jolline I. Forecast verification. A practitioner's Guide in Atmospheric Science. England: Jhon Wiley \& Sons inc. 2003.

[7] Matsudo C, García Y, Ferreiral JR, Salio P, Vidal L, Nicolini M. Sistema de pronóstico experimental en alta resolución con el modelo BRAMS. Meteorológica. 2012; 38(1):53-38.

[8] Maussion FS. WRF simulation of a precipitation event over the Tibetan Plateau, China - An assessment using remote sensing and ground observations. Hydrology and Earth System Sciences, 201 1;15(6), 1795-1817.

[9] Mellor GL and Yamada T. Development of a turbulence closure model for geophysical fluid problems. Rev. Geophys. Space Phys. 1982; 20:851-875. doi:10.1029/RG020i004p00851.

[10] Rubio O, Caucha L, Campos Velho H. Simulación Meteorológica a Nivel Mesoescala del Norte de Perú(2015) usando Brazilian Regional Atmospheric Modelling System (BRAMS). Selecciones Matemáticas. 2015; 2(02):68-75.

[11] Rubio O, Zavaleta J, Carranza A. Sobre la Existencia de Soluciones para un Modelo de Precipitaciones Atmosférica. Selecciones Matemáticas. 2015; 2(01):32-44.

[12] Santos de Oliveira SS, Pereira de Souza EP. Análise de modelos de mesoescala para caracterização do potencial eólico do estado da Paraíba. Revista Brasileira de Meteorologia. 2017; 32(2), 277-291.

[13] Saulo CS, Cardazzo J, Ruiz C, Campetella y Rolla A. El sistema de pronóstico experimental del Centro de Investigaciones del Mar y la Atmósfera. Meteorológica. 2008; 33:83-97.

[14] Thompson G and Eidhammer T. A study of aerosol impacts on clouds and precipitation development in a large winter cyclone. J. Atmos. Sci. 2014; 71:3636-3658. doi:10.1175/JAS-D-13-0305.

[15] Tremback C, Powell J, Cotton W and Pielke R. The forward- in-time upstream advection scheme: Extension to higher orders. Mon. Weather Rev. 1987; 115:540-555.

[16] Tripoli GJ and Cotton WR. The Colorado State University three-dimensional cloud/mesoscale model. Part I: General theoretical framework and sensitivity experiments. J. Rech. Atmos. 1982; 16:185-220.

[17] Wilks DS. Statistical Methods in the Atmospheric Sciences. Second Edition ed. San Diego, California, USA: Elseiver Inc. 2006.

[18] Williams PD. A proposed modification to the Robert-Asselin time filter. Mon. Weather Rev. 2009; 137:2538-2546. 